\title{
The importance of a device specific calibration for smartphone colorimetry
}

\author{
Miranda Nixon ${ }^{1}$, Felix Outlaw ${ }^{1}$, Lindsay W. MacDonald ${ }^{2}$, Terence S. Leung ${ }^{1}$ \\ ${ }^{1}$ Department of Medical Physics and Biomedical Engineering, University College London, UK; ${ }^{2}$ Department of Civil, \\ Environmental \& Geomatic Engineering, University College London, UK
}

\begin{abstract}
In order for a smartphone-based colorimetry system to be generalizable, it must be possible to account for results from multiple phones. A move from device-specific space to a device independent space such as XYZ space allows results to be compared, and means that the link between $X Y Z$ values and the physical parameter of interest needs only be determined once. We compare mapping approaches based on calibration data provided in image metadata, including the widely used opensource software dcraw, to a separate calibration carried out using a colorcard. The current version of dcraw is found to behave suboptimally with smartphones and should be used with care for mapping to XYZ. Other metadata approaches perform better, however the colorcard approach provides the best results. Several phones of the same model are compared and using an xy distance metric it is found that a device-specific calibration is required to maintain the desired precision.
\end{abstract}

\section{Introduction}

There are a wide range of fields within which nonsubjective color measurements are sought. Within medicine, applications include screening for conditions such as jaundice [1, 2], anaemia [3], and anterior blepharitis [4] via images of eye or lower eyelid. Also of interest is the quantification of colorimetric test results, such as urine tests for $\mathrm{pH}$, glucose and protein [5-7], or saliva tests for alcohol concentration [8]. Outside medicine, applications are equally wide-ranging and include testing water quality [9] and comparing color in marine monitoring [10].

Custom devices for measuring color exist, however these may not be appropriate for situations which require non-contact measurements and are often relatively expensive. Smartphones provide an alternative - they are low cost and portable, and better than digital cameras in that they have the capability of carrying out processing in a self-contained way. Smartphone use is becoming ever more prevalent with the global total of subscriptions reaching over 8 billion by 2018 [11], and with access to the technology not limited even in low resource settings. A previous limitation of smartphones was poor image quality and limited access to raw images, however nowadays image quality has exceeded an adequate standard and with the majority of smartphones allowing raw image access.

A major problem in developing a flexible color quantification method is accounting for ambient light. Simple options include calibrating the phone before every measurement [5], including a colorcard in every image [3], or removing the ambient light entirely $[2,8,9]$, however these complicate image capture and may require the development of custom pieces of kit. One solution is to use pairs of flash-no flash images, and perform a subtraction to remove the effects of ambient light, leaving results as though they were captured only under the illumination provided by the phone $[12,13]$. This process enables colors captured using the same phone to be compared across different capture sessions. However, for any colorimetry method to be more generally useful, it must be possible to account for data from different phones. This concept is the focus of this paper, as it is the crucial step necessary for a method to be generalizable, and its impact is often overlooked.

The color values returned by two different phones for images of the same object, even under identical illumination, will be different. This is because the color filters and sensors of phones vary in their spectral sensitivity, meaning that the values recorded are different. The way to enable datasets from different phones to be compared is to move to a device independent space (such as CIEXYZ space), however this step is non-trivial. The typical way to do this is to use a linear mapping from the device space to the general space - it has been found that the additional complication of introducing machine learning does not improve accuracy [14] - however there are different ways to generate and use these mappings. In the context of colorimetry, the goal is to produce values in XYZ space that are precise and reliable such that the link between $X Y Z$ values and the physical parameter of interest need only be developed once. Especially in situations where a ground truth value in XYZ space cannot be measured, it is easy to overlook how large an impact this mapping can have and how imprecise the resulting values can be. It is hard to separate noise coming from real-world data and noise coming from a poor mapping. Therefore, in this paper we use a controlled environment to present and compare two broad categories of approaches for moving to XYZ space - ones based around information provided in the metadata and one based on imaging a colorcard as a one-time calibration step.

\section{Theory}

When using smartphone images to quantify color, it is crucial to use the raw images recorded by the camera. The JPEG images we are used to viewing on our phones have undergone several stages of processing after the values are recorded, including lossy compression and non-linear scaling, to improve the aesthetic result and reduce their size for storage [15]. This means that the values stored are no longer directly related to incident light, and causes huge problems for quantitative measurement. Over the last few years, it has become more and more common to be able to access raw images from smartphone cameras, making more phones viable candidates for quantifying color.

In this paper, we present data captured with an illumination provided by the smartphone flash or white screen with no ambient illumination. This is to enable the impact of the mapping to XYZ space to be considered without any potential error introduced by ambient light. As discussed in the Introduction, the ambient subtraction method using flash-no flash pairs could be used to account for changes in ambient light, meaning that the mappings discussed here would be applicable to data resulting 
from subtraction. Since the data ends up under a standardized illumination, the calibration needs only to be performed once, dramatically simplifying the future image capture process. The smartphone provided illumination was chosen to highlight the ease of extending the ambient subtraction method discussed in the Introduction to multiple phones, however any fixed illumination could equally be considered.

We now focus on how to move from phone native space, where results will vary between phones due to variation in the spectral sensitivity and the spectral power distribution of the flash/screen, to a device independent space, CIEXYZ. Note that we focus on using a matrix mapping to convert RGB to XYZ, and do not consider ICC/DNG profiles owing to particular model-specific metadata not being present in our smartphone metadata and the approaches' need for additional software and equipment, making it hard to integrate with on-phone processing. Here we group the options for the move from native to XYZ space into two broad categories - metadata approaches, which rely only on phone model-specific information stored in the raw image metadata, and a colorcard approach, which utilizes images of a physical colorcard to develop a mapping. Metamerism owing to the Luther condition not being met for the cameras means that the aim is to find the closest approximation for the transformation to XYZ space from native space [16].

\section{Metadata approaches - dcraw}

The first metadata approach we will consider is that of the widely used open-source software dcraw [17]. The method implemented by dcraw utilizes one of two calibration matrices stored in the raw metadata - these matrices map from XYZ space to phone native space, based typically around illuminant D65 and one other standard illuminant. The dcraw implementation uses the D65 calibration matrix, and so the following description refers to D65 for clarity, however the same approach could be used for the other matrix

The overall mapping is achieved by applying the following matrices to the recorded RGB values [18]:

$$
\left[\begin{array}{l}
\mathrm{X} \\
\mathrm{Y} \\
\mathrm{Z}
\end{array}\right]_{\mathrm{D} 65}=\underline{\mathrm{C}}^{-1} \underline{\mathrm{D}}_{\mathrm{D} 65}^{-1} \underline{\mathrm{D}}\left[\begin{array}{l}
\mathrm{R} \\
\mathrm{G} \\
\mathrm{B}
\end{array}\right]_{\text {scene }}
$$

where $\underline{\mathrm{C}}$ is the stored colormatrix moving from $\mathrm{XYZ}$ to native space; $s$ is a scaling factor determined such that the whitepoint of D65 in XYZ [0.9504, 1, 1.0888] is mapped to a native space green channel value of 1 upon application of $\underline{\mathrm{C}}$; and $\underline{\mathrm{D}}$ is a $3 \times 3$ diagonal matrix of white balance multipliers, designed to move the recorded values for a white object to $[1,1,1]$. These multipliers can be obtained through knowledge of the RGB values for white under the scene illumination (in cases of a fixed ambient illumination, the metadata values can be used). D D65 $_{\text {is }}$ another $3 \times 3$ diagonal matrix, this time inverted to move $[1,1,1]$ to the white point of D65 in native space. In other words, the diagonal entries of DD65 $^{-1}$ are simply the white point of D65 in native space. These values can be obtained as follows

$$
\left[\begin{array}{l}
\mathrm{R}(\mathrm{WP}) \\
\mathrm{G}(\mathrm{WP}) \\
\mathrm{B}(\mathrm{WP})
\end{array}\right]=\frac{1}{\mathrm{~s}} \mathrm{C}\left[\begin{array}{l}
\mathrm{X}(\mathrm{WP})=0.9504 \\
\mathrm{Y}(\mathrm{WP})=1 \\
\mathrm{Z}(\mathrm{WP})=1.0888
\end{array}\right]_{\mathrm{D} 65}
$$

where $s$ and $\underline{C}$ are applied to the $X Y Z$ value of the D65 whitepoint to move to native space. Throughout this paper we use a D50 whitepoint, so the final step is to apply a chromatic adaptation transform (CAT) to the data to shift the whitepoint here the Bradford transform was used [18]

\section{Metadata approaches - ForwardMatrix}

Also stored in the image metadata for dng files are what are called 'Forward matrices'. According to the Adobe dng specification this approach behaves better for more extreme values so has been considered here as an alternative to the dcraw approach [19]. These forward matrices convert directly from phone native space to XYZ D50

$\left[\begin{array}{c}\mathrm{X} \\ \mathrm{Y} \\ \mathrm{Z}\end{array}\right]_{\mathrm{D} 50}=\mathrm{M}_{\mathrm{FM}} \underline{\mathrm{D}}\left[\begin{array}{l}\mathrm{R} \\ \mathrm{G} \\ \mathrm{B}\end{array}\right]_{\text {scene }}$

where $\underline{M}_{F M}$ is the forward matrix and $\underline{D}$ is the white balance matrix as before [19]. The metadata includes two forward matrices, taken at high and low color temperatures. If the color temperature of the illumination is known, then an interpolation may be carried out to determine the optimal combination of the two forward matrices. For our case, where the details of the illumination are unknown a simple mean of the two matrices was used.

All metadata methods have the limitation that the transforms were optimized for illuminations other than our scene illumination and that the transform is only provided on a permodel basis rather than a per-device basis, which we will see is crucial.

\section{Colorcard approach}

An alternative approach to using the metadata conversions is to generate a mapping based on a set of known pairs of RGB and XYZ values. Obtaining these sets of values could be achieved in two ways. Option 1: the device could be fully characterised by measuring the spectral power distribution of the flash/screen and the spectral sensitivity of the camera. The reflectances of the surfaces desired for the calibration could be measured, and the RGB values could then be simulated along with producing the $\mathrm{XYZ}$ values. This is undesirable for smartphone calibration as it relies on the user having access to the specialist equipment required to carry out the phone characterization, or introduces error if the characterization is carried out in a more approximate way. Option 2: a physical colorcard could be imaged using the device and illumination of interest to obtain RGB values, and XYZ values measured or the standard provided values used. This option enables the user to calibrate the phone without additional equipment, so we have focused on this method. To generate a standard mapping, the Macbeth ColorChecker Classic card was used, as it is widely available and covers a range of colors yet is small enough to enable a calibration from a single image capture.

Since the illumination provided by a smartphone flash/screen is highly non-uniform, it is necessary to carry out an intensity non-uniformity correction (INUC). This is achieved by capturing a second image of a grey card, with as little movement between captures as possible. A pixel-wise scaling of the colorcard pixel values using the green channel of the grey card image accounts for variations in intensity as follows

$\mathrm{RGB}_{\text {corr }}=\frac{\mathrm{RGB}_{\mathrm{c}}-\mathrm{RGB}_{\mathrm{c}, \text { dark }}}{\mathrm{G}_{\mathrm{w}}-\mathrm{G}_{\mathrm{w}, \mathrm{dark}}}$

where the $c$ subscript refers to pixels in the colorcard image, $w$ refers to the grey card image, and dark refers to images of colorcard and grey card with no illumination. Note that linear, not gamma-encoded, RGB values extracted from the raw image data should be used.

There are many different options for generating the matrix mapping between RGB and XYZ values. The most common approaches in the literature are linear and polynomial mappings. 
We require that the mapping be exposure time independent, to allow images to be taken at different illumination intensity levels without affecting the resulting chromaticities, which rules out polynomial mappings. One option would be a root polynomial mapping [20], however for the number of patches considered here this would likely lead to overfitting. Hence the simplest option, the linear mapping, is selected and calculated

$\mathrm{M}=\left(\mathrm{R}^{\mathrm{T}} \mathrm{R}\right)^{-1} \mathrm{R}^{\mathrm{T}} \mathrm{H}$

where $\mathrm{R}$ and $\mathrm{H}$ are $\mathrm{Nx} 3$ matrices of $\mathrm{RGB}$ and $\mathrm{XYZ}$ values respectively, and $\mathrm{N}$ is the number of patches [18]. Irradiance independent mappings, which do not depend on the relative intensities of the patches, were considered as an alternative to a linear INUC $[20,21]$, however a significant reduction in accuracy was found and so the original linear INUC approach was used.

\section{Methods}

For this study two different models of phone were considered - the Samsung S8 and the LG Nexus 5X, referred to throughout the paper as simply S8 and Nexus. For image capture, the S8 rear camera was used with illumination provided by the LED flash, whereas the front-facing camera of the Nexus was used with a white screen as the illumination. In all cases, images were captured with no ambient illumination. The two phones yielded images of dimensions 3024x4032 and 1944x2592 respectively. Depending on the application, it may be more useful to use either the front or rear-facing camera, hence we considered an example of both. To investigate the variability of these phones within a specific model, two devices of each model were used. The linearity of the response of each phone to incident light was verified before use.

148 patches from the Macbeth ColorChecker DC card were used for testing the mapping approaches (excluding the repeating neutrals from the boundary of the chart, the reflective patches and those out of the gamut of the Classic card). The patch reflectances were measured using the $\mathrm{x}$-rite ColorMunki spectrophotometer and the resulting ground truth xy values are shown in Figure 1 as yellow crosses. The card was imaged in sections with each phone to enable a reasonable illumination and regions of interest selected. All mapping approaches considered are exposure time independent so it did not matter that the intensity of light varied across the images. At the scale of a single patch, the intensity was uniform hence the resulting xy chromaticities were unaffected by the variation.

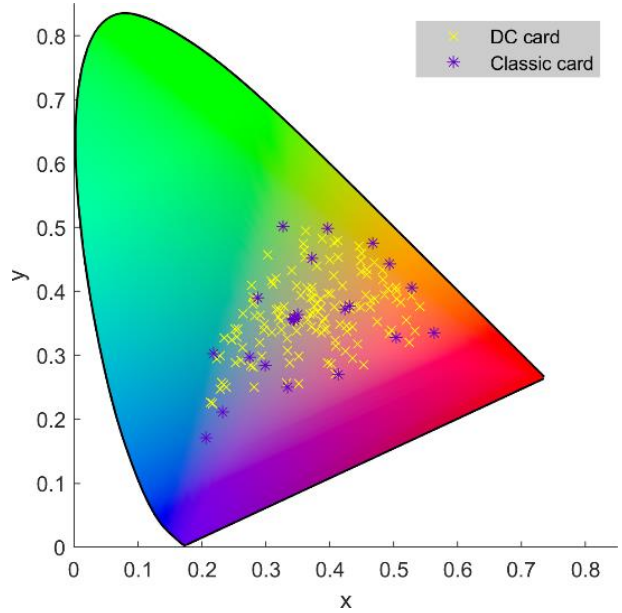

Figure 1. The ground truth xy values for the Macbeth $D C$ card used for testing (yellow crosses) are shown along with the ground truth xy values for the Macbeth Colorchecker Classic card used for the colorcard approach (purple stars).
For the first metadata approach, dcraw (Dave Coffin version $9.27,2016)$ was used to process the images to 16-bit linear tiff images in XYZ space, using pre-measured white balance multipliers. Additionally, the process used by dcraw was implemented in MATLAB (MathWorks r2018a) to verify details of the method. The ForwardMatrix metadata approach was also implemented in MATLAB. The patch xy values could then be extracted directly from the processed images.

For the colorcard approach, the Macbeth ColorChecker Classic 24 patch card was used. The patch reflectances were again measured using the $\mathrm{x}$-rite ColorMunki spectrophotometer, and the xy values are shown in Figure 1 as purple stars. Images of the card were captured for each phone using the phone illumination with no ambient light. Here the relative patch values are crucial so in order to account for the varying illumination intensity provided by the phone, an intensity non-uniformity correction (INUC) was carried out as described in the Theory section. Once the INUC had been performed, it was then possible to construct the RGB to XYZ mapping using the extracted RGB values and measured $\mathrm{XYZ}$ values. This mapping was applied to native RGB values extracted from the test images to obtain $x y$ values.

\section{Performance Metric}

It should be noted that the method presented here provides only xy information, not full XYZ values. For many colorimetry applications, precise $x y$ values are all that is required and the increased flexibility of not having to include anything additional in test images outweighs the negative of losing the third dimension. The method could be extended to produce full XYZ values through the inclusion of a white standard in each image to normalize for lightness, but this was deemed unnecessary here. The lack of full XYZ values means that the typical $\Delta \mathrm{E}$ error metric in $\mathrm{L}^{*} \mathrm{a} \mathrm{b}^{*}$ space cannot be used. This space is specifically designed to be more perceptually uniform and the benchmark $\Delta \mathrm{E}$ values are in reference to human vision. An alternative approach would be to consider not $x y$ chromaticity values but $u^{\prime} v^{\prime}$ values which are a simple transform away from xy values, from the CIE 1976 uniform chromaticity scale diagram which is designed to be more perceptually uniform [23]. The $u^{\prime} v^{\prime}$ distance between predicted and ground truth values could then be used as a performance measure. We selected XYZ space as it is a standard device independent color space, not because of its relation to human vision. The aim of this research is not to mechanize human color judgements but rather to obtain repeatable digital color descriptors that can then be linked to the relevant physical biomarker scale. In alignment with previous literature in this area, xy chromaticity space was used $[5,6]$ and the xy distance between mapped and ground truth patch values was therefore used to quantify performance of the different methods

$\mathrm{xy}$ distance $=\sqrt{\left(\mathrm{x}_{\mathrm{M}}-\mathrm{x}_{\mathrm{GT}}\right)^{2}+\left(\mathrm{y}_{\mathrm{M}}-\mathrm{y}_{\mathrm{GT}}\right)^{2}}$

where subscript M and GT refer to mapped and ground truth chromaticity values respectively. The overall goal is for the results from different phones to match each other and so if they are found to match the ground truth then this goal is achieved.

\section{Results and Discussion}

The first method considered was the metadata approach using dcraw. Figure 2 shows the resulting xy values scattered with the ground truth values for one Nexus and one S8. Upon visual inspection of the overall distribution, the match between 
measured and ground truth xy values seemed worse than expected, especially given how widely used and trusted dcraw is.

Upon implementing the dcraw method in MATLAB it was discovered that in line with a mention in the literature [18], dcraw makes the assumption that the colormatrix for D65 illumination is always stored as the second colormatrix in the metadata. Whilst this may well be the case for digital cameras, by considering the CalibrationIlluminant 1 and 2 metadata tags, it was found that for these smartphone models the D65 calibration matrix is in fact the other matrix. Figure 3 provides a visual description of how the choice of colormatrix differs between dcraw and when knowledge of the calibration illuminant is included. It is little surprise that dcraw produces poor results as the implementation is incorrect here. Use of dcraw to XYZ space with smartphones should therefore be used with care.

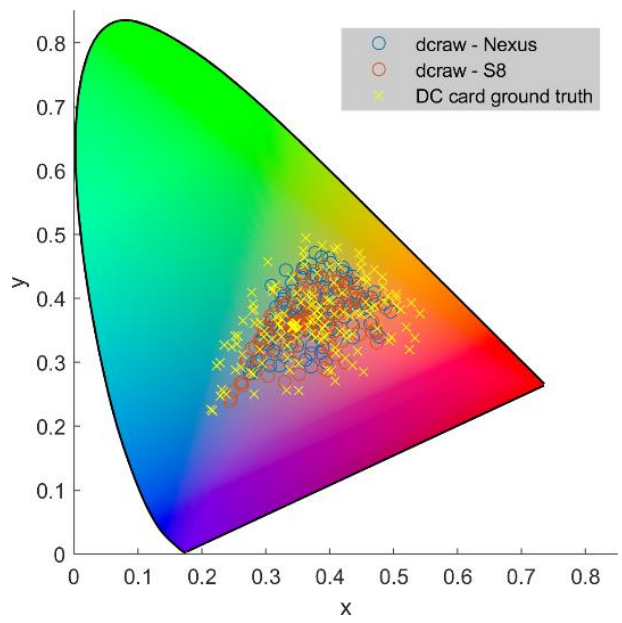

Figure 2. Ground truth $x y$ values for the DC card (yellow crosses) along with the values produced by dcraw processing of images from a Nexus (blue circles) and an S8 (red circles).

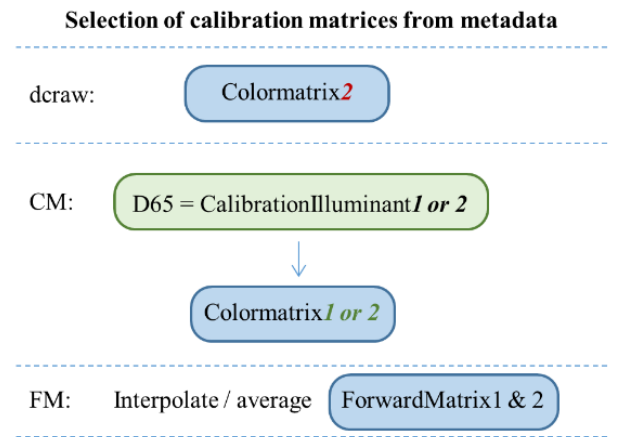

Figure 3. Visual description of the choice of calibration matrices from the metadata for dcraw, CM (correct colormatrix) and FM (forward matrix) methods. Note that dcraw does not use the calibration illuminant tags to select the colormatrix but uses a default.

Figure 4 shows the accuracy of the four approaches for a Nexus and an S8 - similar trends were obtained for the phones of a given model across the different approaches, so the results for an example phone of each model are shown here. To calculate the data shown in Figure 4, the following steps were followed for each phone and mapping:

- Find the distance of each mapped patch to the ground truth

- For a given radial threshold xy distance $(0-0.1)$

- Find the number of mapped patches which fall within this radial value of their respective ground truth
This data then presents the likely accuracy of the mapping method for a given desired xy distance uncertainty. Note that the trends seen in Figure 4 were identical when using $u^{\prime} v^{\prime}$ distances.

From an inspection of Figure 4, it is clear that for very high xy distance thresholds, or in other words for discriminating between very different colors, it does not matter which mapping is chosen as all give good results. As the xy distance decreases the dcraw results drop off particularly quickly. For both phones, as expected, the use of the correct colormatrix greatly improves the dcraw-style metadata approach. For the Nexus phones, the ForwardMatrix approach behaves similarly to the ColorMatrix approach, whereas there is a significant improvement for the S8 when using the ForwardMatrix. Since the overall goal is to find a calibration method which enables different phones to be used together, the ForwardMatrix method is clearly not a reliable option since it produces such poor results for the Nexus phone.

The colorcard approach outperforms the metadata approaches at almost all levels, particularly for low threshold xy distances. Therefore, in order to achieve xy values of a usefully precise level, it is necessary to carry out a calibration stage using images of a colorcard.

(a)

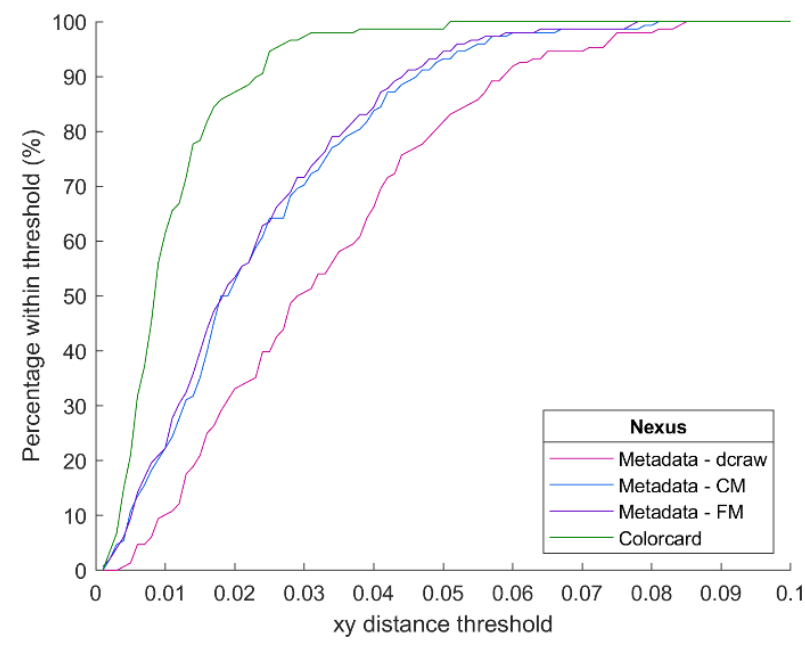

(b)

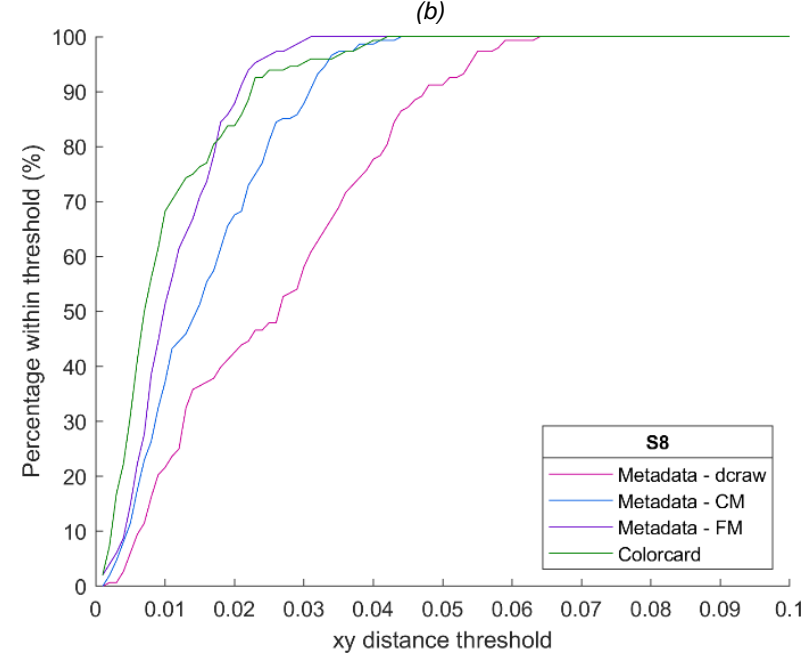

Figure 4. The percentage of patches falling within different $x y$ distances are shown in (a) and (b) for a Nexus and an S8 phone respectively. The results are shown for four different mapping options: dcraw (pink), dcraw-style using the correct colormatrix (blue), ForwardMatrix (purple), colorcard (green). For low target $x y$ distances, the colorcard approach provides the best results for both phone models. 
At this stage, it would be tempting to assume that the colorcard mapping developed for a phone of a given model would be applicable to another phone of the same make and model. It is known that variations in digital cameras prohibit this $[15,21]$, however results for smartphones have not been presented. Unfortunately, the combination of subtle variations in the filters and sensors combined with variations in the spectral power distributions of the LEDs or screens of typical smartphones is too great to allow a model-level calibration. Figure 5 shows a demonstration of the impact using the wrong mapping can have, where the mapping developed using one Nexus phone is applied to the other Nexus. A similar reduction in accuracy was observed for all phones, hence just the Nexus has been included as an example.

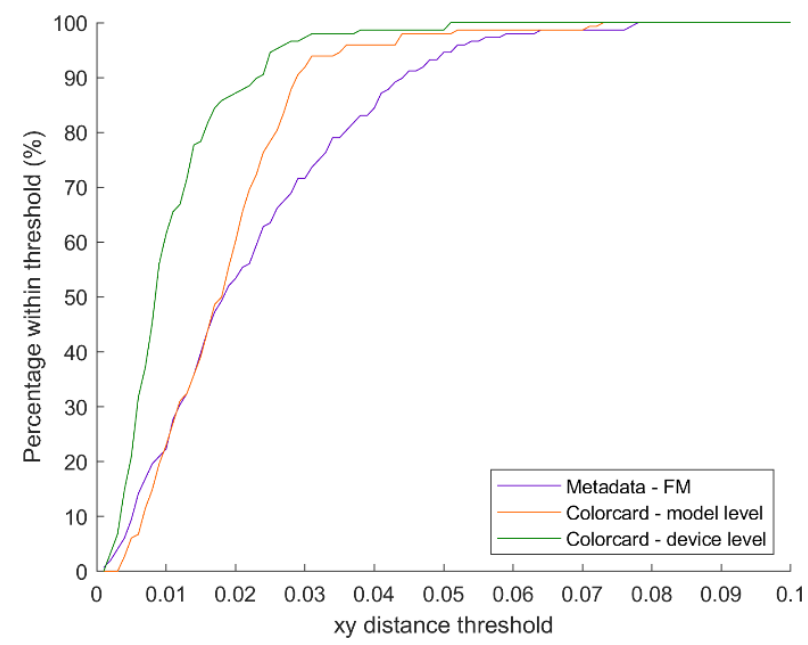

Figure 5. The percentage of patches falling within different $x y$ distances is shown for a Nexus phone (similar results for S8 phones), contrasting the precision between a device-level calibration and a model-level calibration. The ForwardMatrix metadata method is shown in purple, and the colorcard approach mapping developed using the same device is shown in green. The result of using a colorcard mapping developed using a different Nexus phone is shown in orange. The significant loss in accuracy especially for small $x y$ distances highlights the need for a device specific calibration to maintain accuracy.

When a model-level mapping is applied, the loss of accuracy incurred for low xy distances is so great that the colorcard approach is reduced in quality to the metadata approaches. For example, at an xy threshold of 0.01 the percentage falls from $61 \%$ to just $23 \%$. To achieve the desired higher level accuracies, it is therefore vital that phones are calibrated on a per-device basis. Whilst this may sound unrealistic for making a colorimetric method more widely available, the calibration needs only to be carried out once per device. Since this mapping is intended to be used in combination with an ambient subtraction approach, the illumination is standardized to the phone illumination after subtraction despite varying ambient light. And when the per-device colorcard approach is used, data from different phones become combinable.

\section{Conclusions}

In this paper, we have showcased the ability of smartphones to provide compatible chromaticity measurements between phones of different makes when calibrated correctly, using a simple one-time calibration and ambient subtraction. We have highlighted the requirement that the development of a mapping from images of a colorcard is carried out on a per-device basis in order to achieve the best accuracy. The negative impact of using a per-model level calibration is such that if phones are not going to be individually calibrated then it is no longer worth using the colorcard method and instead a metadata approach should be used, with the exception of using dcraw which is unreliable for conversion to XYZ with smartphones. In fact, it should be noted that the unreliability of dcraw for conversion to $\mathrm{XYZ}$ is a more general issue for smartphones, relevant regardless of the approach for dealing with ambient light. If the full $\mathrm{XYZ}$ values are required, then it is possible to extend the method presented here through the inclusion of a white standard in each image allowing the lightness information to be recovered. However, when xy values are all that is required the colorcard calibration method presented here allows data from different phones to be combined. This means that the more complicated task of finding a link between xy values and the physical scale of interest need only be carried out once.

\section{References}

[1] T. S. Leung, F. Outlaw, L. W. MacDonald, and J. Meek, "Jaundice Eye Color Index (JECI): quantifying the yellowness of the sclera in jaundiced neonates with digital photography," Biomed. Opt. Express, vol. 10, no. 3, p. 1250, Mar. 2019.

[2] A. Mariakakis, M. A. Banks, L. Phillipi, L. Yu, J. Taylor, and S. N. Patel, "BiliScreen: SmartphoneBased Scleral Jaundice Monitoring for Liver and Pancreatic Disorders," Proc. ACM Interactive, Mobile, Wearable Ubiquitous Technol., vol. 1, no. 2, pp. 1-26, 2017.

[3] S. Collings, O. Thompson, E. Hirst, L. Goossens, A. George, and R. Weinkove, "Non-invasive detection of anaemia using digital photographs of the conjunctiva," PLoS One, vol. 11, no. 4, 2016.

[4] V. Y. Bunya et al., "Assessment of signs of anterior blepharitis using standardized color photographs," Cornea, vol. 32, no. 11, pp. 1475-1482, 2013.

[5] A. K. Yetisen, J. L. Martinez-Hurtado, A. GarciaMelendrez, F. Da Cruz Vasconcellos, and C. R. Lowe, "A smartphone algorithm with inter-phone repeatability for the analysis of colorimetric tests," Sensors Actuators, B Chem., vol. 196, pp. 156-160, Jun. 2014.

[6] L. Shen, J. A. Hagen, and I. Papautsky, "Point-of-care colorimetric detection with a smartphone," Lab Chip, vol. 12, no. 21, pp. 4240-4243, 2012.

[7] A. Y. Mutlu, V. Kiliç, G. K. Özdemir, A. Bayram, N. Horzum, and M. E. Solmaz, "Smartphone-based colorimetric detection via machine learning," Analyst, vol. 142, no. 13, pp. 2434-2441, 2017.

[8] Y. Jung, J. Kim, O. Awofeso, H. Kim, F. Regnier, and E. Bae, "Smartphone-based colorimetric analysis for 
detection of saliva alcohol concentration," Appl. Opt., vol. 54, no. 31, p. 9183, 2015.

[9] V. Kiliç, G. Alankus, N. Horzum, A. Y. Mutlu, A. Bayram, and M. E. Solmaz, "Single-Image-Referenced Colorimetric Water Quality Detection Using a Smartphone," ACS Omega, vol. 3, no. 5, pp. 55315536, May 2018.

[10] D. Akkaynak, E. Chan, J. J. Allen, and R. T. Hanlon, "Using spectrometry and photography to study color underwater," Ocean. Mts/Ieee Kona, pp. 1-8, Sep. 2011.

[11] I. T. Union, "Mobile cellular subscriptions data," 2018. [Online]. Available:

http://data.worldbank.org/indicator/IT.CEL.SETS/coun tries?display=default.

[12] J. M. DiCarlo, F. Xiao, and B. a Wandell, "Illuminating Illumination," Ninth Color Imaging Conf., no. January 2001, pp. 27-34, 2001.

[13] C. Lu and M. S. Drew, "Practical Scene Illuminant Estimation via Flash / No-Flash Pairs," Fourteenth Color Imaging Conf., no. January 2006, pp. 84-89, 2006.

[14] C. Vien, W. Stephen, C. David, and R. Caterina, “A comparative study of the characterisation of colour cameras by means of neural networks and polynomial transforms," Color. Technol., vol. 120, no. 1, pp. 1925, 2004.

[15] D. Akkaynak et al., "Use of commercial off-the-shelf digital cameras for scientific data acquisition and scene-specific color calibration," J. Opt. Soc. Am. A, vol. 31, no. 2, p. 312, 2014.
[16] R. Luther, "Aus dem Gebiet der Farbreizmetrik (On color stimulus metrics)," Zeitschrift für Tech. Phys., vol. 8, no. 1927, pp. 540-558, 1927.

[17] D. Coffin, "Decoding raw digital photos in Linux." [Online]. Available: https://www.cybercom.net/ dcoffin/index.html. [Accessed: 05-Mar-2019].

[18] A. Rowlands, S. D. J. Gwyn, H. Wang, K. Lee, and J. Ryu, Chapter 4 Raw conversion. IOP Publishing, 2017.

[19] Adobe Systems Incorporated, "Digital Negative (DNG) specification. Version 1.4.0.0," 2012.

[20] G. D. Finlayson, M. MacKiewicz, and A. Hurlbert, "Color Correction Using Root-Polynomial Regression," IEEE Trans. Image Process., vol. 24, no. 5, pp. 1460-1470, May 2015.

[21] B. Funt and P. Bastani, "Irradiance-independent camera color calibration," Color Res. Appl., vol. 39, no. 6, pp. 540-548, Dec. 2014.

[22] P. Bastani and B. Funt, "Simplifying irradiance independent color calibration," in Color Imaging XIX: Displaying, Processing, Hardcopy, and Applications, 2014, vol. 9015, p. 90150N.

[23] R. W. G. Hunt and M. R. Pointer, Measuring Colour. Chichester, UK: John Wiley \& Sons, Ltd, 2011.

[24] T. E. White, R. L. Dalrymple, D. W. A. Noble, J. C. O'Hanlon, D. B. Zurek, and K. D. L. Umbers, "Reproducible research in the study of biological coloration," Anim. Behav., vol. 106, pp. 51-57, Aug. 2015. 